\title{
O CAMPO DA GESTÃO DO CONHECIMENTO A PARTIR DE BIBLIOMETRIAS
}

\section{THE KNOWLEDGE MANAGEMENT FIELD FROM BIBLIOMETRIES}

\author{
Maria Ligia Ganacim Granado Rodrigues Eliasa \\ Hilka Pelizza Vier Machadob
}

\begin{abstract}
RESUMO
Introdução: A Gestão do Conhecimento é um campo interdisciplinar, em decorrência disso, diversos estudos bibliométricos sobre o campo foram publicados, procurando identificar sua abrangência e contornos. Objetivo: Mapear estudos bibliométricos em Gestão do conhecimento, a fim de compreender as fronteiras do campo de estudo, bem como as interfaces com outras áreas. Metodologia: Qualitativa, constituindo-se em uma análise sistemática de estudos bibliométricos sobre gestão do conhecimento entre os anos de 2008 e 2018. Resultados: Os estudos bibliométricos demonstraram que o campo surgiu por volta dos anos 1970 e se consolidou a partir de algumas obras seminais. Ao longo da década de 1990 diversas áreas contribuíram com a abordagem de temáticas que foram incorporadas aos estudos de GC. A partir dos anos 20000 número de publicações expandiu consideravelmente, tornando o campo multifacetado e desenvolvendo-se em duas vertentes, uma com publicações científicas em diversos Journals e outra em Conferências. Conclusões: O campo da GC apresenta-se como um campo amplo de estudos, com a contribuição de seguintes áreas: Ciência da Computação, Administração/Gestão/Organizações; Economia; Ciência da Informação/Sistemas de Informação; Comunicação; Ciência da Computação, Inteligência Artificial e Biblioteconomia. Há pouca convergência entre as áreas que contribuem para a construção do campo, tanto em termos de autores, como em publicações.
\end{abstract}

Descritores: Gestão do Conhecimento. Bibliometria. Informação. Produção acadêmica.

\footnotetext{
a Doutora em Ciência Política pela Universidade de São Paulo (USP). Pós Doutoranda Capes no Programa de Pós Graduação em Gestão do Conhecimeno nas Organizações, Centro Universitário Maringá (UniCesumar). E-mail: ligiagranado@gmail.com

b Doutora em Engenharia de Produção pela Universidade Federal de Santa Catarina (UFSC). Pesquisadora do Instituto Cesumar de Inovação, Ciência e Tecnologia. Professora do Doutorado em Administração da Universidade do Oeste de Santa Catarina (Unoesc). E-mail: hilkavier@yahoo.com
} 


\section{INTRODUÇÃO}

O campo da Gestão do Conhecimento (GC) foi construído por profissionais de mercado, incumbidos de desenvolver o capital intelectual em empresas. Por volta dos anos 1990, o campo conquistou reconhecimento (MA; YU, 2010; SERENKO et al., 2011). O número de estudos em GC cresceu gradualmente ao longo dos anos subsequentes. Contudo, persiste um consenso entre os autores sobre a dificuldade em compreender os limites do campo e sua identidade (SERENKO, 2013).

A GC é, desde o início, um campo interdisciplinar (KOKOL et al. 2015, QIU; LV, 2014; SERENKO, 2013). Além disso, outra característica do campo é a publicação de apenas um artigo pela maioria dos autores, evidenciando que a área não tem definida uma seleção de tópicos que definam exclusivamente os seus limites (SERENKO et al. 2011; WANG et al., 2018).

Em decorrência disso, diversos estudos bibliométricos sobre GC foram publicados, procurando identificar a abrangência e os contornos do campo. Estudos bibliométricos podem ser utilizados para fazer análises qualitativas e, segundo Wallin (2005, p. 261), "o maior objetivo dos exercícios bibliométricos é transformar algo intangível (qualidade científica) em algo mensurável". Essas análises estão firmemente estabelecidas como uma metodologia de avaliação de campos de estudos (ELLEGAARD; WALLIN, 2015).

Considerando que os estudos bibliométricos no campo da GC são diversos e que utilizam diferentes bases de dados e períodos, constata-se que isoladamente eles não são capazes de apresentar um panorama abrangente da área e apenas uma análise conjunta desses estudos é capaz de demonstrar a abrangência do campo de estudos. Com isso, o problema que este estudo busca investigar é: Como se configura a área de GC a partir do conjunto de bibliometrias? Quais os autores mais influentes? Quais áreas têm interfaces de estudos? O estudo justifica-se na medida em que, apesar dos quase quarenta anos de estudos, a GC carece de uma ontologia comum e de uma base teórica comum, que propicie uma clara direção de pesquisa (SERENKO, 2013). 
Com isso, esta pesquisa tem como objetivo geral mapear estudos bibliométricos em Gestão do Conhecimento, a fim de compreender as fronteiras do campo, bem como as interfaces com outras áreas e principais autores. Como objetivos específicos pretende-se, a partir dos estudos bibliométricos, identificar autores influentes, publicações e interfaces com outras áreas.

Para atingir o objetivo, realizou-se uma revisão sistemática de literatura sobre bibliometrias em GC. Por meio dessa revisão, analisou-se um conjunto de estudos publicados nos últimos dez anos. Em seguida, os estudos foram analisados quanto a autores que fundamentaram o campo, publicações e interfaces com outras áreas. Os resultados propiciam um panorama evolutivo do campo e podem auxiliar pesquisadores, estudantes e profissionais que atuam ou desejam se inserir na área. Para o contexto brasileiro o estudo é importante para estimular o debate sobre o campo da GC, ainda pouco presente.

\section{CONSIDERAÇÕES SOBRE CIENTOMETRIA E BIBLIOMETRIA}

Estudos bibliométricos são importantes para avaliar a ciência e o fluxo de informação (CASSUNDÉ; BARBOSA; MENDONÇA, 2018) e têm por objetivo "transformar algo intangível (qualidade científica) em algo mensurável" (WALLIN, 2005, p. 261). A análise bibliométrica corresponde a uma análise quantitativa de publicações científicas (ELLEGAARD, WALLIN, 2015), cujo foco é analisar o desenvolvimento passado, presente e futuro da ciência (SERENKO, 2013). Trata-se de um tipo de pesquisa que têm levado ao desenvolvimento de diferentes métricas para iluminar a estrutura intelectual de uma disciplina acadêmica e avaliar o impacto de periódicos científicos, estudos e pesquisadores (AKHAVAN et al., 2016; KOKOL et al., 2015). Estudos bibliométricos fazem parte da cientometria, que por sua vez pode ser entendida como uma "ciência sobre a ciência" e também como um campo acadêmico que tem estabelecido linhas de perguntas, metodologias e uma identidade distinta (RAMY et al., 2018, p. 2). Cientometria pode ser definida também como "a métrica da ciência, um estudo de mensuração e quantificação do processo científico baseado em indicadores 
bibliométricos" (SILVA, BIANCHI, 2011, p. 6). Esses estudos visam tanto estabelecer normas, regras e heurísticas para garantir o progresso da disciplina, como também, observar e relatar as atividades realizadas em um campo acadêmico (SERENKO et al., 2010).

A origem desses estudos remonta ao século dezenove (SERENKO, 2013), especificamente em 1874 (quando foi realizada uma survey por 180 cientistas britânicos interessados na influência de suas publicações). No entanto, foi em 1964 que esses estudos passaram a ser disseminados, com a introdução do índice de citação (KOKOL et al., 2015). Serenko (2013) menciona o papel de James Cattell, Editor da Science, que implementou medição sistemática de publicações e que introduziu duas dimensões de mensuração que são atualmente utilizadas, a qualitativa (julgamento por pares) e quantitativa (produtividade). O termo cientomético foi inventado por Nalimov (um matemático russo, em 1969) e, em 1978 foi lançado o primeiro número do Journal Scientometrics, o que contribuiu para reconhecimento acadêmico desse tipo de estudos (SERENKO, 2013).

A finalidade de estudos cientométricos é a de mensurar, classificar e descrever os produtos da ciência, identificar o impacto teórico e prático dos estudos acadêmicos, explorar a natureza de publicações científicas, determinar a alocação mais eficiente de recursos para maximizar pesquisas e seu impacto e propor recomendações para o desenvolvimento de políticas de pesquisa (SERENKO, 2013). Entre as análises utilizadas em estudos bibliométricos destacam-se as análises relacionais, ou seja, análises de co-citação e de acoplamento bibliográfico (MA; YU, 2010). Outras análises comumente empregadas são as de co-ocorrência de palavras-chave e a análise de copalavras que mapeia a força de associação entre os elementos de informação em dados textuais e pode ser temática, semântica, conceitual e cognitiva (GAVIRIA-MARIN; MERIGO; POPA, 2018). Em síntese, esses estudos apresentam uma visão sobre a evolução de publicações, tipos de documento, número de citações, artigos mais citados, autores, instituições e países influentes (WANG et al., 2018).

Evidencia-se assim, que a cientometria, por meio de estudos 
bibliométricos corresponde a uma abordagem quantitativa da produção de um determinado campo, por meio de análises estatísticas e matemáticas. Para esta pesquisa, entende-se que estudos bibliométricos do campo de GC fornecem elementos para a compreensão da formação e do desenvolvimento do campo. Se por um lado justifica-se este recorte, por outro, é pertinente atentar para as limitações deste tipo de estudo, Wang et al. (2018), destacam que foge ao escopo dos estudos bibliométricos questões mais substantivas associadas a teorias, epistemologia e ontologia. A utilização deste tipo de pesquisa para o campo da GC é justificável uma vez que é um campo em constante evolução e o conjunto dos estudos bibliométricos pode propiciar a compreensão do seu desenvolvimento e perspectivas futuras enquanto disciplina autônoma.

\section{PROCEDIMENTOS METODOLÓGICOS}

A revisão sistemática da literatura foi realizada em seis etapas, como mostrado na Figura 1. A revisão sistemática emprega elevado rigor científico, por meio de uma recolha exaustiva dos textos publicados sobre o tema que se pretende analisar (CASSUNDÉ; BARBOSA; MENDONÇA, 2018). Para esta revisão sistemática foram selecionadas as bases de dados: Scopus, Web of Science e Scielo. As bases Scopus e Web of Science são bases cientificamente reconhecidas (ELLEGAARD; WALLIN, 2015). A base de dados Scielo, por sua vez, representa uma das principais bases aberta da América Latina (MUGNAINI; DIGIAMPIETRI; MENA-CHALCO, 2014).

Como um dos critérios de busca, estabeleceu-se artigos científicos publicados em língua inglesa, por ser predominante em publicações da área (RAMY et al., 2018; SERENKO; BONTIS, 2013). Como termos de busca, foram utilizados utilizados os seguintes: knowledge management and citation analysis and citation study and scientometric study and scientometric analysis and scholarly impact. Na base Scopus foram encontrados 85 artigos, na Scielo 258 e na Web of Science 90, totalizando 433 artigos.

O recorte temporal foi o de uma década: de 2008 a 2018, entendendo que pela natureza dos textos estudados: cientometrias/bibliometrias, seus achados 
englobariam estudos publicados anteriormente e ao mesmo tempo, ofereceriam um quadro atualizado de estudos contemporâneos da GC. As buscas foram realizadas no mês de maio de 2019, e a Figura 1 apresenta o passo a passo para seleção dos artigos e os critérios de inclusão e exclusão.

Figura 1 - Seleção dos artigos analisados (critérios de inclusão e exclusão)

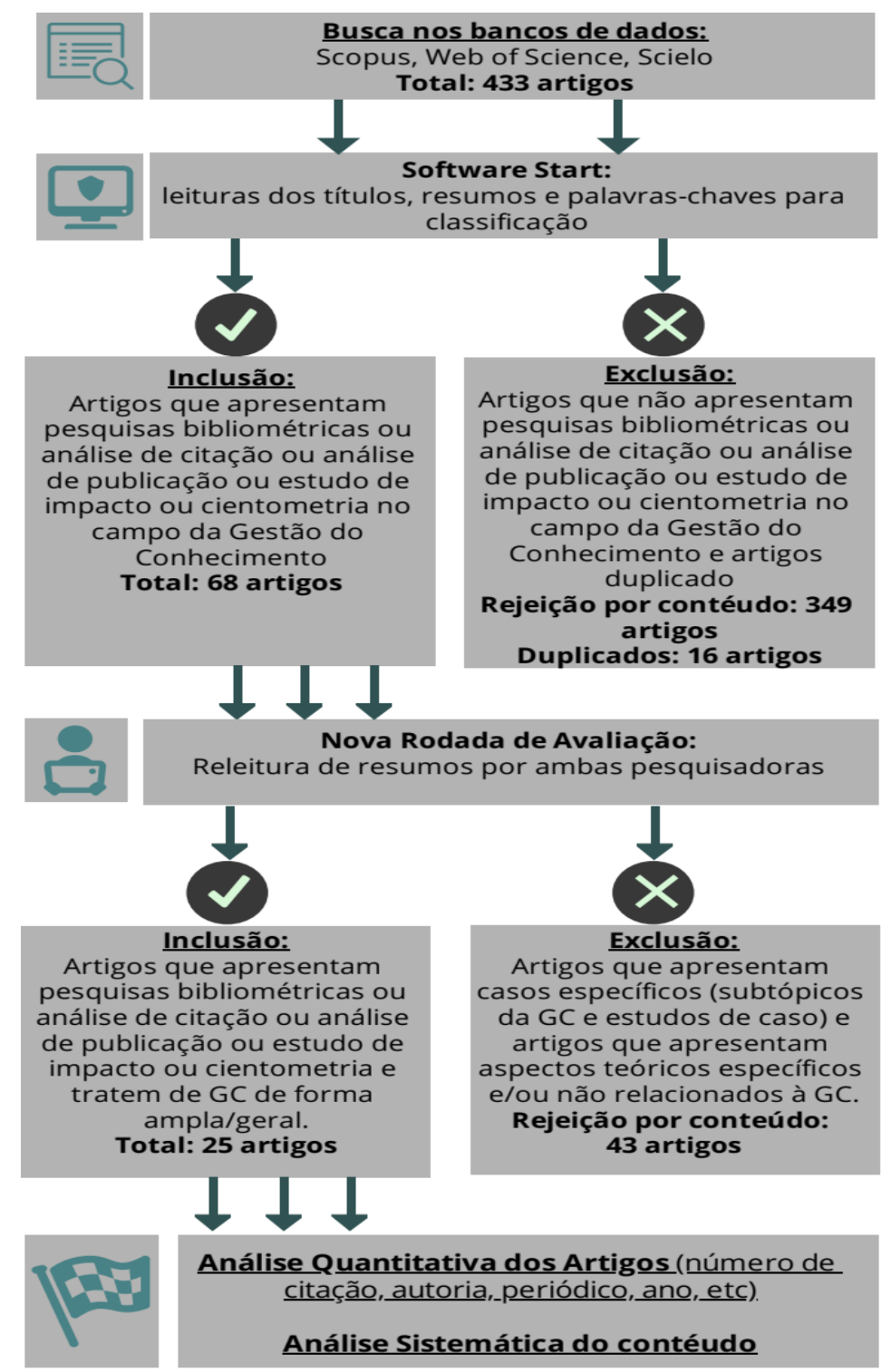

Fonte: Elaboração das autoras

Para exportação dos artigos, foi utilizado o software START. Na sequência, foram extraídas informações, por meio da leitura dos títulos, resumos e palavras-chave. Como critério de inclusão, foram selecionados os artigos que abordassem Bibliometria ou Análise de citação ou Análise de Publicação ou 
Estudo de impacto ou Cientometria combinados com Gestão do Conhecimento. Nessa etapa foram selecionados 68 artigos, representando $16 \%$ do total. Foram excluídos os artigos em duplicidade (16) e os que não abordavam os temas em conjunto (349). Em seguida, foi realizada a leitura dos resumos dos 68 artigos selecionados por ambas as pesquisadoras. Foram excluídos artigos que tratavam de estudos bibliométricos de temas específicos da GC, tendo sido selecionados 25 artigos, dos quais 24 foram publicados em periódicos científicos e um apresentado em congresso. Em nova avaliação, constatou-se que o estudo de Heimerl et al. (2016) não contribuía com os resultados e foi retirado, restando 24 artigos. O artigo de autoria de Serenko e Bontis (2017) foi utilizado em substituição à versão anterior, publicada com resultados até 2012. Em seguida, buscou-se no Google acadêmico o número de citações de cada artigo selecionado. Além disso, buscou-se dados sobre os fatores de impacto das revistas (JCR de 2018). A análise dos dados foi quantitativa, com os dados compilados por meio de tabelas, gráficos e figuras.

\section{RESULTADOS}

O Quadro 1 apresenta um resumo de cada um dos 24 estudos que serviram de base para as análises sistemáticas.

\section{Quadro 1 - Síntese dos estudos bibliométricos}

\begin{tabular}{l|l|l}
\hline Autores & Ano & Síntese do estudo \\
\hline $\begin{array}{l}\text { Bontis; } \\
\text { Serenko. }\end{array}$ & 2009 & $\begin{array}{l}\text { Objetivo de desenvolver um ranking de Journals in GC e capital } \\
\text { intelectual usando H index e g index. Identificaram que as três obras } \\
\text { mais citadas em GC e IC são livros. Os seis journals mais citados da } \\
\text { área são: Journal of Knowledge Management, Journal of Intelectual } \\
\text { Capital, The Learning organization, Knowledge and Process } \\
\text { Management, Knowledge Management Research and Practice, Journal } \\
\text { of Knowledge Management Practice. }\end{array}$ \\
\hline Ma; Yu. 2010 & $\begin{array}{l}\text { O estudo faz uma análise de citação, co-citação entre outros de artigos } \\
\text { publicados entre 1998 e 2007, utilizando o string "Knowledge } \\
\text { management". Base de dados usada: Science Citation Index (SCI); } \\
\text { Social Science Citation Index (SSCI). Coletou 1.230 artigos com 29.601 } \\
\text { publicações citadas como referência. Os autores identificaram dois } \\
\text { momentos do desenvolvimento do campo, um até 2002 e outro de 2002 } \\
\text { a 2007. }\end{array}$ \\
\hline $\begin{array}{l}\text { Serenko et } \\
\text { al. } 2010\end{array}$ & $\begin{array}{l}\text { Os autores realizaram um estudo bibliométrico em 2.175 artigos dos } \\
11 \text { maiores periódicos dos temas Gestão do Conhecimento e Capital } \\
\text { Intelectual entre 1994 e 2008. Constataram que publicações de práticos } \\
\text { são relevantes na área, mas estão reduzindo. }\end{array}$ \\
\hline
\end{tabular}




\begin{tabular}{|c|c|c|}
\hline $\begin{array}{l}\text { Serenko et } \\
\text { al. }\end{array}$ & 2011 & $\begin{array}{l}\text { Buscaram o efeito Matthew em GC e IC (modelo estocástico) a partir da } \\
\text { análise de } 2175 \text { artigos publicados em } 11 \text { journals. Constataram o } \\
\text { predomínio de acadêmicos sobre práticos e a não existência do efeito } \\
\text { Matthew em GC/IC, na medida em que há mais autores que publicam } \\
\text { apenas um paper do que artigos concentradas em poucos acadêmicos, } \\
\text { como seria o efeito. }\end{array}$ \\
\hline $\begin{array}{l}\text { Wallace, } \\
\text { Van Fleet, } \\
\text { Downs }\end{array}$ & 2011 & $\begin{array}{l}\text { Os autores realizaram um estudo bibliométrico e uma análise de } \\
\text { conteúdo em } 630 \text { artigos publicados em } 20 \text { journals em GC. Eles } \\
\text { utilizaram o método Bradford analysis. Eles identificaram como temas } \\
\text { não explorados as consequências negativas de tecnologias } \\
\text { organizacionais no aprendizado. }\end{array}$ \\
\hline $\begin{array}{l}\text { Uzunboylu, } \\
\text { Eris, } \\
\text { Ozcinar }\end{array}$ & 2011 & $\begin{array}{l}\text { Os autores realizaram um estudo bibliométrico em GC na área de } \\
\text { Educação, utilizaram a base de dados da Scopus e identificaram } 125 \\
\text { documentos, publicados entre } 1990 \text { e } 2008 \text {, abrangendo } 39 \text { países. }\end{array}$ \\
\hline $\begin{array}{l}\text { Dwivedi et } \\
\text { al. }\end{array}$ & 2011 & $\begin{array}{l}\text { Os autores realizaram uma meta análise de } 34 \text { anos de publicações em } \\
\text { GC, no periodo } 1974 \text { a } 2008 \text { na base da ISI Web of Science. Analisaram } \\
\text { os } 250 \text { artigos mais recentes, publicados em } 385 \text { diferentes journals. } \\
\text { Utilizaram diversos métodos: bibliometria + análise histórica + meta- } \\
\text { análise. Identificaram seis categorias: ambiente, processos, sistemas, } \\
\text { planejamento, políticas, avaliação e estratégia; pesquisa e educação e } \\
\text { outras. }\end{array}$ \\
\hline $\begin{array}{l}\text { Curado, } \\
\text { Oliveira, } \\
\text { Maçada }\end{array}$ & 2011 & $\begin{array}{l}\text { Examinaram publicações em Gestão do Conhecimento em } 6 \text { journals: } \\
\text { Information And Management, Information Processing And } \\
\text { Management, International Journal Of Information Management, } \\
\text { Journal Of Global Information Management, Journal Of Management } \\
\text { Information Systems, Management Information Systems Quarterly. } \\
\text { Constataram que o nível intra organizacional de análise é pouco } \\
\text { explorado. }\end{array}$ \\
\hline Grant & 2011 & $\begin{array}{l}\text { álise bibliométrica da GC no período de } 1990 \text { a 2009, com base nos } \\
\text { dos do Proquest e em } 1800 \text { artigos de jornais, revistas. O autor } \\
\text { alizou uma análise de conteúdo e identificou um período inicial de } \\
80 \text { a 1994, marcado pelas ideias dos precursores, seguido de um } \\
\text { ríodo de rápido crescimento de } 1995 \text { a 2001, mas um conflito entre } \\
\text { áticos e pesquisadores. }\end{array}$ \\
\hline Lee; Chen. & 2012 & $\begin{array}{l}\text { Bibliométrico busca mapeamento intelectual da área de GC, realizado } \\
\text { com estudos publicados entre os anos de } 1995 \text { a } 2010 \text {. Os autores } \\
\text { analisaram } 10974 \text { papers, dividiram em } 3 \text { períodos: } 1995-2000 ; 2001- \\
2005 \text { e } 2006-2010 \text {. Como base de dados utilizaram Microsoft academic } \\
\text { search, Web of Science, km/IC journals. Utilizaram o PFNet para } \\
\text { identificar interpelação entre temas. }\end{array}$ \\
\hline Serenko. & 2013 & $\begin{array}{l}\text { Meta análise realizada com } 108 \text { estudos cientométricos de GC. } \\
\text { Constatou que a área está em progresso rumo a maturidade. Identificou } \\
\text { três momentos de estudos cientométricos: inicial (1997-2001); } \\
\text { Desenvolvimento inicial (2002-2006) e rigor e consolidação (2007- } \\
\text { 2012). }\end{array}$ \\
\hline $\begin{array}{l}\text { Madeira; } \\
\text { Vick; } \\
\text { Nagano. }\end{array}$ & 2013 & $\begin{array}{l}\text { Analisaram a relação entre Gestão do conhecimento, gestão da } \\
\text { inovação, gestão de tecnologia e gestão da informação. Selecionaram } \\
\text { publicações entre } 2006-2012 \text { em } 10 \text { journals, um total de } 295 \text { artigos. }\end{array}$ \\
\hline $\begin{array}{l}\text { Serenko; } \\
\text { Bontis }\end{array}$ & 2013 & $\begin{array}{l}\text { Análise cientometrica no Journal of Knowledge Management, com H- } \\
\text { index. Analisaram } 63 \text { artigos citados } 5.939 \text { vezes. Ressaltam a } \\
\text { participação de livros e artigos de práticos e apresenta fragilidade na } \\
\text { identidade, pois se apropria de conhecimentos de outras áreas. }\end{array}$ \\
\hline $\begin{array}{l}\text { Walter; } \\
\text { Ribière. }\end{array}$ & 2013 & $\begin{array}{l}\text { O estudo faz análise de citação e co-citação das publicações do } \\
\text { Knowledge Management Research and Practice entre julho de } 2003 \text { e } \\
\text { set de } 2012 \text { (256 artigos). Os } 100 \text { artigos mais citados estão agrupados } \\
\text { em quatro tópicos"; a) comunidades e aprendizado; b) networks, }\end{array}$ \\
\hline
\end{tabular}

Inf. Inf., Londrina, v. 25, n. 1, p. $320-344$, jan./mar. 2020. 


\begin{tabular}{|c|c|c|}
\hline & & $\begin{array}{l}\text { transferência de conhecimento e métodos de pesquisa; c) fundamentos } \\
\text { da área de GC e d) capital intelectual. }\end{array}$ \\
\hline Qiu; Lv. & 2014 & $\begin{array}{l}\text { Análise bibliométrica da GC na web of Science de } 1993 \text { a } 2012 \text {. } \\
\text { Identificaram } 12925 \text { publicações, sendo } 6996 \text { de proceedings e } 4312 \\
\text { artigos. Artigos representaram } 42 \% \text { da produção e livros } 41,04 \% \text {. . }\end{array}$ \\
\hline Kokol et al. & 2015 & $\begin{array}{l}\text { Utilizou a base da Scopus e analisou publicações entre 1996-2010 } \\
\text { utilizando os termos knowledge management and organization. No total } \\
\text { foram consultadas } 10.599 \text { fontes de informação, incluindo conferencias } \\
\text { (5111), artigos (4337), revisões (496), capítulos de livro (352), livros } \\
\text { (86), editorial e outros. Os autores identificaram quatro tópicos } \\
\text { principais: Educação e cuidado da saúde, técnicas e sistemas, gestão } \\
\text { do conhecimento e compartilhamento do conhecimento. } \\
\text { Cronologicamente os autores identificaram cinco momentos: pesquisa } \\
\text { organizacional e infraestrutura, questões técnicas e sistemas de gestão } \\
\text { conhecimento em organizações, tecnologias, aplicações práticas da } \\
\text { gestão do conhecimento em organizações, e Avaliação e validação de } \\
\text { práticas de gestão do conhecimento. }\end{array}$ \\
\hline $\begin{array}{l}\text { Khasseh; } \\
\text { Mokhtarpour }\end{array}$ & 2016 & $\begin{array}{l}\text { Busca identificar historicamente os artigos mais citados em GC por meio } \\
\text { da análise de citação utilizando um novo método de análise } \\
\text { cientometrica - "References Publication Years Spectroscopy (RPYS)" } \\
\text { - que analisa as oscilações de publicação ao longo dos anos. Esse } \\
\text { método procura identificar as contribuições históricas e os trabalhos que } \\
\text { tiveram importância na formação da área, para isso foram analisadas } \\
\text { quais foram as referências bibliográficas usadas nos artigos. Foram } \\
\text { analisados artigos publicados no "Thomsom citation index" (artigos de } \\
1980-2014 \text { ), como o foco são as referências históricas foram analisadas } \\
\text { àquelas entre os anos de } 1900 \text { até } 1980 \text {. Os autores identificaram a } \\
\text { influência das áreas de Administração, Economia, Redes Sociais, } \\
\text { Aprendizado organizacional e sociologia econômica. }\end{array}$ \\
\hline $\begin{array}{l}\text { Akhavan et } \\
\text { al. }\end{array}$ & 2016 & $\begin{array}{l}\text { Bibliométrico dos } 500 \text { artigos mais citados no campo de GC, entre } 1980 \\
\text { e 2014, tendo como base de dados a Web of Science Para evitar viés } \\
\text { nas citações os autores fizeram um index (não pegaram os números } \\
\text { brutos). O index foi baseado no número de citações por ano. } \\
\text { Analisaram } 3198 \text { artigos. }\end{array}$ \\
\hline $\begin{array}{l}\text { Serenko; } \\
\text { Bontis. }\end{array}$ & 2017 & $\begin{array}{l}\text { Atualização do estudo bibliométrico realizado anteriormente pelos } \\
\text { autores no período } 2008 \text { a } 2012 \text {. Excluíram os seguintes journals que } \\
\text { saíram de circulação: act KM: Online Journal of KM, último número em } \\
\text { 2009; Open Journal of Knowledge Management - último número em } \\
\text { 2012; Journal of Knowledge Management Practice, último número em } \\
\text { 2013. Utilizaram as seguintes bases de dados: Ulrich's Periodical } \\
\text { Directory, Google Scholar, Google Search Engine. Usaram apenas os } \\
\text { que não aparecem na lista de publicações predatórias da Beall. } \\
\text { Excluíram Journal of organizational Knowledge Management; Intangible } \\
\text { capital; Journal of Knowledge Management, Economics and information } \\
\text { Technology; Knowledge and Performance Management; International } \\
\text { Journal of Data Mining and Knowledge Management Process. }\end{array}$ \\
\hline Wang et al. & 2018 & $\begin{array}{l}\text { Objetivo geral é avaliar o status de pesquisa em GC e identificar as } \\
\text { características da área por meio da análise bibliométrica das palavras } \\
\text { chave. Selecionaram artigos da web of Science entre } 1974-2017 \text {, um } \\
\text { número total de } 7628 \text {. Análise de frequência de palavras-chave sendo } \\
\text { que } 835 \text { principais palavras-chave com uma frequência maior ou igual } \\
\text { a cinco foram escolhidas para análise de rede de co-ocorrência } \\
\text { utilizando o VOSviewer, Citespace, Carrote Bib Excel. }\end{array}$ \\
\hline $\begin{array}{l}\text { Gaviria- } \\
\text { Marin; } \\
\text { Merigo; } \\
\text { Popa. }\end{array}$ & 2018 & $\begin{array}{l}\text { Realizaram um bibliométrico do Journal of Knowledge Management. - } \\
\text { Análise das publicações } 1997 \text { e } 2016 \text { desenvolvendo o que os autores } \\
\text { chamam de análise de performance e mapeamento científico, variáveis } \\
\text { analisadas: Acoplamento bibliográfico, co-citação, co-palavras, co- } \\
\text { ocorrência de palavras-chaves ou redes. }\end{array}$ \\
\hline
\end{tabular}

Inf. Inf., Londrina, v. 25, n. 1, p. 320 - 344, jan./mar. 2020. 


\begin{tabular}{l|l|l}
\hline & \multirow{2}{*}{$2018 \begin{array}{l}\text { Bibliometria dos artigos publicados no Journal of Information \& } \\
\text { Knowledge Management (JIKM) entre 2002-2016, analisando 475 } \\
\text { artigos publicados neste jornal a partir da taxonomia de Tan et al. } \\
\text { (2009). Utiliza analise de palavras-chaves como também análise de } \\
\text { conteúdo. Identificaram volume crescente de publicação e tópicos } \\
\text { Alhaji. } \\
\text { como: descoberta do conhecimento, taxonomia e ontologia, } \\
\text { representação do conhecimento e tendência para estudos quantitativos } \\
\text { por meio de questionários. }\end{array}$} \\
\hline Ramy et al. $2018 \begin{array}{l}\text { Cientometria do Knowledge Management Research \& Practice } \\
\text { (KMRP) entre os anos de 2003-2015. Constataram que a colaboração } \\
\text { entre autores aumentou, mas que alguns setores de conhecimento } \\
\text { intensivo ainda não estão sendo explorados em pesquisas. É crescente } \\
\text { também o foco em artigos empíricos, mais do que em revisões de } \\
\text { literatura. }\end{array}$ \\
\hline $\begin{array}{l}\text { Goswami; autores realizaram um estudo bibliométrico na WOS sobre } \\
\text { publicações em compartilhamento do conhecimento e identificaram 11 } \\
\text { journals líderes e 190 artigos. Utilizaram os softwares Bib Excel e } \\
\text { VOSviewer e identificaram quatro temas principais: perspectiva } \\
\text { orientada pelo comportamento, compreensão, frameworks e modelos, } \\
\text { perspectiva orientada pela tecnologia e barreiras no compartilhamento. }\end{array}$ \\
\hline
\end{tabular}

Fonte: Elaboração das autoras

Em relação ao ano de publicação das bibliometrias, verifica-se que um estudo foi publicado em 2009, dois em 2010, seis em 2011, um em 2012, quatro em 2013, um em 2014, um em 2015, dois em 2016, um em 2017 e cinco em 2018. No Quadro 2 observa-se o número de citações, autoria e periódico de publicação, com respectivo fator de impacto. A bibliometria mais citada (289) foi a de Serenko et al., (2010), seguida de Serenko e Bontis (2017), com 149 citações. Ambas foram publicadas no Journal of Knowledge Management. No entanto, o Journal que apresenta maior fator de impacto é o International Journal of Information Management (5.063), mas este estudo publicado em 2011 recebeu apenas 74 citações. É importante ressaltar a publicação de Akhavan et al. (2016), no Journal Scientometrics, focado em bibliometrias, citado 87 vezes. Somente no ano de 2018 foram publicadas cinco bibliometrias, no entanto, como elas foram publicadas recentemente é difícil concluir o impacto, pois as citações recentes tendem a ser menos referenciadas (AKHAVAN et al., 2016).

\section{Quadro 2 - Citação e fator de impacto dos artigos selecionados}

\begin{tabular}{cccc}
\hline Autores & Citação & Journal & $\begin{array}{c}\text { FI } \\
\text { revistas } \\
\text { (2018) }\end{array}$ \\
\hline Wang et al., 2018 & 7 & Sustainability & 2.592 \\
\hline $\begin{array}{c}\text { Gaviria-Marin; } \\
\text { Merigo; Popa, 2018 }\end{array}$ & 9 & Journal of Knowledge Management & 4.604 \\
\hline
\end{tabular}




\begin{tabular}{|c|c|c|c|}
\hline Alajmi; Alhaji, 2018 & 2 & $\begin{array}{c}\text { Journal of Information \& Knowledge } \\
\text { Management }\end{array}$ & - \\
\hline Ramy et al., 2018 & 5 & Knowledge Management Research \& Practice & 1.485 \\
\hline $\begin{array}{l}\text { Goswami; Agrawal, } \\
2018 \\
\end{array}$ & 2 & VINE Journal of Information and KM Systems & - \\
\hline $\begin{array}{l}\text { Serenko; Bontis, } \\
2017\end{array}$ & 149 & Journal of Knowledge Management & 4.604 \\
\hline $\begin{array}{c}\text { Khasseh; } \\
\text { Mokhtarpour, } 2016\end{array}$ & 12 & Journal of Knowledge Management & 4.604 \\
\hline $\begin{array}{c}\text { Akhavan et al., } \\
2016\end{array}$ & 87 & Scientometrics & 2.770 \\
\hline Kokol et al., 2015 & 5 & $\begin{array}{c}\text { International Conference on K. M in } \\
\text { Organizations KMO } 2015\end{array}$ & - \\
\hline Qiu; Lv, 2014 & 28 & Aslib Journal Of Information Management & 2.12 \\
\hline Serenko, 2013 & 83 & Journal of Knowledge Management & 4.604 \\
\hline $\begin{array}{l}\text { Madeira; Vick; } \\
\text { Nagano, } 2013\end{array}$ & 6 & Transinformação & 0.2830 \\
\hline $\begin{array}{l}\text { Serenko; Bontis, } \\
2013\end{array}$ & 79 & Journal of Knowledge Management & 4.604 \\
\hline $\begin{array}{c}\text { Walter; Ribière, } \\
2013\end{array}$ & 24 & Knowledge Management Research \& Practice & 1.485 \\
\hline Lee; Chen, 2012 & 130 & Knowledge-Based Systems & 5.101 \\
\hline $\begin{array}{l}\text { Serenko et al., } \\
2011\end{array}$ & 43 & Journal of Infometrics & 3.878 \\
\hline $\begin{array}{l}\text { Wallace; Van Fleet; } \\
\text { Downs, } 2011\end{array}$ & 74 & International Journal of Information Management & 5.063 \\
\hline $\begin{array}{l}\text { Uzunboylu; Eris; } \\
\text { Ozcinar, } 2011\end{array}$ & 20 & British Journal of Educational Technology & 2.588 \\
\hline Dwivedi et al., 2011 & 112 & Information Systems Management & 2.042 \\
\hline $\begin{array}{l}\text { Curado; Oliveira; } \\
\text { Maçada, } 2011\end{array}$ & 12 & African Journal Of Business Management & - \\
\hline Grant, 2011 & 9 & $\begin{array}{c}\text { Electronic Journal of Knowledge Management: } \\
\text { EJKM }\end{array}$ & - \\
\hline $\mathrm{Ma} ; \mathrm{Yu}, 2010$ & 131 & Journal of Knowledge Management & 4.604 \\
\hline $\begin{array}{l}\text { Serenko et al., } \\
2010\end{array}$ & 289 & Journal of Knowledge Management & 4.604 \\
\hline $\begin{array}{l}\text { Bontis; Serenko } \\
2009\end{array}$ & 120 & Journal of Knowledge Management & 4.604 \\
\hline
\end{tabular}

Fonte: Elaboração das autoras

No Gráfico 1 explicita-se o número de citações por artigo recebidas até junho de 2019. Autores como Serenko e Bontis destacam-se tanto em suas cooperações, quanto em relação ao número de citações recebidas. Outros autores como Ma, Yu (2010), Lee, Chen (2012) e Dwivedi et al. (2011) receberam mais de cem citações cada um. 


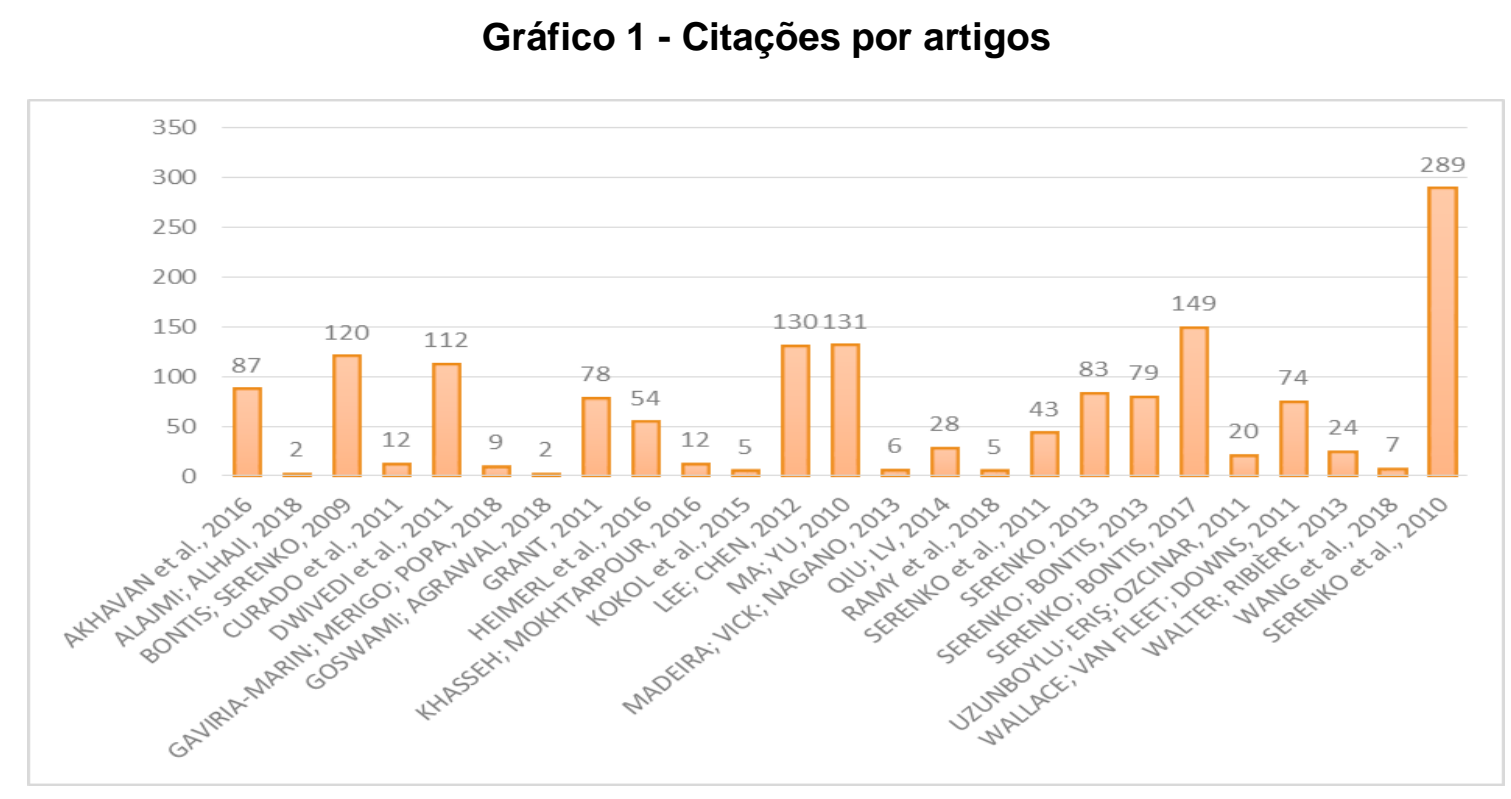

Fonte: Elaboração das autoras. Citações até 06/2019.

\subsection{Avaliação do Campo de GC por Meio dos Estudos Bibliométricos}

A avaliação do campo centrou-se em três aspectos: os periódicos da área, os autores mais citados e a relação com outras disciplinas.

\subsubsection{Periódicos Científicos Centrais Para a Área de GC}

Os periódicos com maior representatividade na área foram: (JKM) Journal of Knowledge Management, citado 12 vezes nos 25 artigos; (KMRP) Knowledge Management Research \& Practice, citado 9 vezes; International Journal of Information Management e The Learning Organization, ambos citados 5 vezes. No Gráfico 2 destacam-se os periódicos que foram citados mais de uma vez e que são vistos como estruturantes do campo de GC. Ao lado de cada periódico constam os artigos em que o título foi identificado, evidenciando que a avaliação de periódicos ocorreu em 17 dos textos analisados. 


\section{Gráfico 2 - Eixo temático - Periódicos que estruturam o campo - número de citações nos artigos analisados}

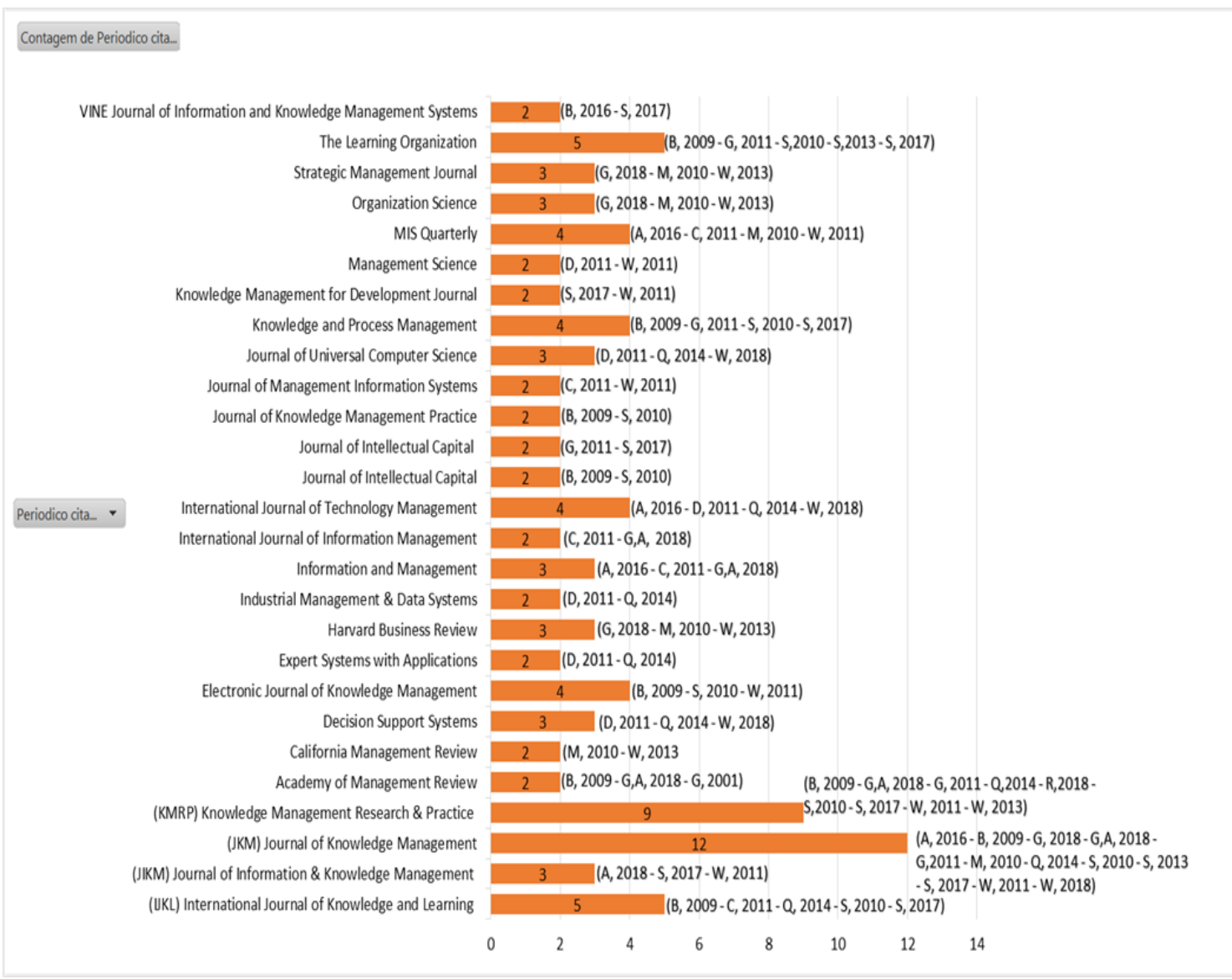

Legenda: AKHAVAN et al., 2016 - A, 2016; ALAJMI; ALHAJI, 2018 - A, 2018; BONTIS; SERENKO, 2009 - B, 2009; CURADO; OLIVEIRA, MAÇADA, 2011 - C, 2011; DWIVEDI et al., 2011 - D, 2011; GAVIRIA-MARIN; MERIGO; POPA, 2018 - G, 2018; GOSWAMI; AGRAWAL, 2018 - G, A, 2018; GRANT, 2011 - G, 2011; MA; YU, 2010 - M, 2010; QIU; LV, 2014 - Q, 2014; RAMY et al., 2018 - R, 2018; SERENKO et al., 2010 - S, 2010; SERENKO; BONTIS, 2013 - S, 2013; WALLACE; VAN FLEET; DOWNS, 2011 - W, 2011; WALTER; RIBIĖRE, 2013 - W, 2013; WANG et al., 2018 - W, 2018.

É praticamente um consenso entre os autores que o Journal of Knowledge Management é o mais representativo da área, sendo o mais citado (GAVIRIAMARIN et al. 2018; KOKOL et al., 2015; QIU; LV, 2014; SERENKO et al. 2010), exceto para Alajami e Alhaji, (2018), para os quais o Journal of Information and Knowledge Management foi o mais citado, e para Dwivedi et al. (2011), que identificaram o maior número de publicações no International Journal of Technology Management.

O segundo Journal mais citado é o Knowledge Management Research \& 
Practice, exceto para Serenko (2013) que identificou o International Journal of Knowledge Management e o Knowledge \& Process Management. Nesse sentido, não há um consenso entre os estudos analisados sobre o grau de importância de outros periódicos, além do Journal of Knowledge Management. Por exemplo, Bontis e Serenko (2009) identificaram os cinco mais citados, depois do Journal of Knowledge Management: Journal of Intellectual Capital, The Learning organization, Knowledge and Process Management, Knowledge Management Research and Practice, Journal of Knowledge Management Practice. Por outro lado, Kokol et al. (2015) citaram como o segundo mais citado - Vine, seguido de: Journal of Information and Knowledge Management; Communication in Computer and Information Science, Knowledge of Management Research and Practice, Learning Organizations. De modo semelhante Alajmi e Alhaji (2018) mencionaram o terceiro mais citado, o Knowledge Management Research and Practice, seguido do Journal of Intellectual Capital, International Journal of Learning and Intellectual Capital, Knowledge and Process Management, Communication in Computer and Information Science, Vine, Information Sciences, Journal of the Operational Research Society, Journal of Theoretical and Applied Information technology and Learning Organization. Por sua vez, Gaviria-Marin, Merigo e Popa (2018) consideram que os Journals mais importantes foram: Knowledge Management Research and Practice, Vine, Journal of Information and Knowledge Management, Journal of Intellectual Capital, International Journal of Knowledge Management, Knowledge and Process Management, Learning Organization e International Journal of Knowledge Based Development. Já Serenko et al. (2010) identificaram, além do Journal of Knowledge Management, os seguintes: Learning Organization, Knowledge and Process Management, Journal of Intellectual capital, Electronic Journal of Knowledge Management, Knowledge Management Research \& Practice, International Journal of Learning and Intellectual Capital.

Outra ordem de importância dos periódicos foi apontada por Ma e Yu (2010), cujo bibliométrico utilizou as bases SCI e SSCI, sendo os Journals mais citados em ordem: Organization Science, Harvard Business Review, Strategic 
Management Journal, California Management Review, MIS Quarterly, Sloan Management Review, Journal of Knowledge Management, Academy of Management Review, Administrative Science Quarterly, Decision Support Systems, Management Science, Journal of Management Information Systems, Academy of Management Journal and Expert Systems with Applications. Este último (Expert Systems with Applications) e o Knowledge Management Research \& Practice foram mencionados também por Qiu e Lv (2014) e por Wang et al. (2018). Além destes, Wang et al. (2018) identificaram como periódicos importantes: International Journal of Technology Management; Decision Support Systems; Journal of Universal Computer Science, International Journal of Information Management, Industrial Management Data Systems, Information Management, Knowledge-based Systems, Journal of Computer Information Systems, Journal of Information Science, Knowledge Management Research \& Practice, Lecture Notes in Computer Science, Lecture Notes in Artificial Intelligence. Para Goswami e Agrawal (2018), além do Journal of Knowledge Management os mais importantes foram os journals: Organization Science e MIS Quarterly, Academy of Management Journal e Academy of Management Review.

Com base nos dados, o Journal of Knowledge Management é a principal publicação do campo. Também é possível identificar Journals restritos ao campo como: Knowledge Management Research \& Practice; International Journal of Knowledge Management; Knowledge \& Process Management; Electronic Journal of Knowledge Management e International Journal of Knowledge-Based Development. Os demais enquadram-se em três áreas do conhecimento, sendo Informação/Comunicação, Sistemas/Tecnologias e Gestão/Estratégia, como mostrados no Quadro 3.

\section{Quadro 3 - Distribuição dos Journals por área}

\begin{tabular}{lll}
\hline Informação/Comunicação & Gestão/Estratégia & Sistemas/Tecnologias \\
\hline $\begin{array}{l}\text { Journal of information and } \\
\text { Knowledge Management }\end{array}$ & Journal of Intellectual Capital & $\begin{array}{l}\text { International Journal of } \\
\text { Technology Management }\end{array}$ \\
\hline $\begin{array}{l}\text { Communication in Computer } \\
\text { and Information Science }\end{array}$ & Learning Organization & $\begin{array}{l}\text { Vine Journal of information } \\
\text { and Knowledge Management } \\
\text { Systems }\end{array}$ \\
\hline $\begin{array}{l}\text { Journal of computer and International Journal of } \\
\text { Information Systems }\end{array}$ & $\begin{array}{l}\text { Learning and Intellectual } \\
\text { Capital Support Systems }\end{array}$ \\
\hline
\end{tabular}




\begin{tabular}{|c|c|c|}
\hline $\begin{array}{lll}\begin{array}{l}\text { Journal of } \\
\text { Science }\end{array} & & \\
\end{array}$ & Organization Science & $\begin{array}{lll}\text { Journal of } & \text { Universal } \\
\text { Computer Science } & \\
\end{array}$ \\
\hline $\begin{array}{lr}\text { Journal of } & \text { Theoretical and } \\
\text { Applied } & \text { Information } \\
\text { Technology } & \end{array}$ & Harvard Business Review & $\begin{array}{l}\text { Lectures Notes in Computer } \\
\text { Science }\end{array}$ \\
\hline Information Management & $\begin{array}{l}\text { Strategic } \\
\text { Journal }\end{array}$ & $\begin{array}{l}\text { Lectures Notes in Artificial } \\
\text { Intelligence }\end{array}$ \\
\hline $\begin{array}{l}\text { Journal of Management } \\
\text { Information Systems }\end{array}$ & $\begin{array}{ll}\text { California } & \text { Managem } \\
\text { Review } & \\
\end{array}$ & Knowledge-Based Systems \\
\hline \multirow[t]{3}{*}{$\begin{array}{lcc}\text { International Journal of } \\
\text { Information Management }\end{array}$} & $\begin{array}{l}\text { Sloan Management Review } \\
\text { Journal }\end{array}$ & MIS Quarterly \\
\hline & $\begin{array}{l}\text { Academy of Management } \\
\text { Review }\end{array}$ & $\begin{array}{l}\text { Industrial Management Data } \\
\text { Systems }\end{array}$ \\
\hline & $\begin{array}{l}\text { Administrative } \\
\text { Quarterly }\end{array}$ & $\begin{array}{l}\text { Expert Systems } \\
\text { Applications }\end{array}$ \\
\hline
\end{tabular}

Fonte: Elaboração das autoras

\subsubsection{Autores Importantes na Formação do Campo}

O autor que maior influência exerceu nos fundamentos filosóficos do campo da GC foi Polanyi, que em 1966 publicou a obra The tacit dimension (KHASSEH; MOKHTARPOUR, 2016; QIU; LV, 2014). Para Polanyi o julgamento humano não se limita ao nível coletivo de significados historicamente construídos, mas manifesta-se também no nível individual, o que implica que "todo conhecimento é um conhecimento pessoal" (TSOUKAS; WLADIMIROU, 2001, p. 981). A concepção de conhecimento tácito foi o ponto central da teoria do conhecimento desenvolvida posteriormente por Nonaka e Takeuchi (1995).

Conforme alguns autores (DWIVEDI et al., 2011; GAVIRIA-MARIN; MERIGO; POPA, 2018; QIU; LV, 2014; WANG et al., 2018; GOSWAMI; AGRAWAL, 2018; SERENKO, 2013), as três obras mais referenciadas no campo foram Nonaka e Takeuchi (1995), Davenport e Prusak (1998) e Nonaka (1994). Para Ma Yu (2010) eles foram mais citados no período 1998 a 2007. Nesse sentido, as obras de Nonaka e Takeuchi (1995), Davenport e Prusak (1998) e Nonaka (1994) foram as que maior influência exerceram para a formação do campo, sendo os três, livros. Outro autor importante nesse período de desenvolvimento do campo foi Wiig (1997), mencionado por Gaviria-Marin, Merigo, Popa (2018) e Serenko (2013). Outros autores mencionam estudos sobre capacidade absortiva e sobre teoria baseada no conhecimento e Cohen e Levinthal (1990) e Grant (1996) estão entre os mais citados (GAVIRIA-MARIN; 
MERIGO; POPA, 2018; QIU; LV, 2014). No campo das organizações, a abordagem do conhecimento é influenciada pela obra de Peter ZUPIC (1994), como comentaram Ramy et al. (2018).

No início da década de 2000, autores do campo de sistemas de informação começam a publicar no campo e o estudo de Alavi e Leidner (2001) está entre os mais citados (GAVIRIA-MARIN; MERIGO; POPA, 2018; LEE; CHEN, 2012; QIU; LV, 2014). Além destes, outro tema que se insere na agenda de estudos é o capital intelectual, sendo Sveiby e Martins (2006) o autor mais citado (GAVIRIA-MARIN; MERIGO; POPA, 2018; SERENKO, 2013). Entre os estudos mais citados com perspectiva crítica destaca-se o de Wilson (2002), intitulado "The nonsense of knowledge management" (SERENKO, 2013).

No entanto, quando se consideram publicações em conferências os autores mais citados no período 1995 e 2000, segundo Lee e Chen (2012), foram: Gruber, com 1294 citações (Toward principles for the design of ontologies used for knowledge sharing), seguido de Guarino, com 894 citações (Formal ontology and information systems). Partindo do pressuposto que as conferências englobam estudiosos e práticos, observa-se uma falta de convergência entre os autores citados, evidenciando a presença duas vertentes distintas de produção de conhecimento. Esse aspecto precisa ser considerado, pois as conferências representam aproximadamente $50 \%$ das publicações no campo (KOKOL et al., 2015). Além disso, os estudos bibliométricos mais recentes pontuam como crítico o fato de muitos autores publicarem no campo, mas não de forma contínua, evidenciando que a opção por pesquisa em GC constitui uma opção secundária de pesquisa (SERENKO, 2013). Por exemplo, Wang et al (2018) identificaram que 14.808 autores publicaram três artigos ou menos cada um e Dwivedi et al. (2011) identificaram que entre 2019 autores que publicaram na área, 1796 publicou apenas um artigo cada. Também Serenko et al. (2010) mencionaram que $80 \%$ dos autores publicaram apenas uma vez.

\subsubsection{Relação da GC com Outras Disciplinas}

De acordo com os estudos consultados, as publicações em GC ocorreram em diversas áreas. Dwivedi et al. (2011) identificaram 89 áreas e Wang et al. 
(2018) trinta áreas de estudos. Entre os temas em palavras chave dos 500 artigos mais citados, Akhavan et al. (2016) identificaram: conhecimento, gestão, organização, aprendizado e sistemas.

As disciplinas centrais que mostram a interdisciplinaridade do campo são: Ciência da Computação, Administração/Gestão/Organizações; Economia; Ciência da Informação/Sistemas de Informação; Comunicação; Ciência da Computação, Inteligência Artificial e Biblioteconomia (AKHAVAN et al., 2016; ALAJMI; ALHAJI, 2018; DWIVEDI et al., 2011; KHASSEH; MOKHTARPOUR, 2016; KOKOL et al., 2015; WANG et al., 2018). Qiu e Lv (2014) consideram que a principal área de interface é a Gestão, que concentrou $31,58 \%$ das publicações, seguida de Ciência da Computação e Sistemas de Informação, que juntas representaram 28,02 \% das publicações, seguidas de Ciência da Informação e Biblioteconomia, com 18,26 \% das publicações.

Identifica-se também uma interface com outras áreas, como a Engenharia (AKHAVAN et al. 2016; ALAJMl; ALHAJI, 2018; KOKOL et al., 2015). Wang et al. (2018) especificam as Engenharias Industrial, de Software, Elétrica e Civil. Outras áreas que mencionaram estes estudos foram: Psicologia e Ciências Cognitivas (AKHAVAN et al., 2016; ALAJMI; ALHAJI, 2018; KHASSEH; MOKHTARPOUR, 2016; WANG et al. 2018); Pesquisa operacional (AKHAVAN et al., 2016; WANG et al. 2018); Contabilidade (KOKOL et al., 2015); Marketing e Sociologia Econômica (KHASSEH; MOKHTARPOUR, 2016).

Além disso, autores identificaram publicações de GC nas áreas de Saúde e Médica (AKHAVAN et al., 2016; ALAJMI; ALHAJI, 2018; WANG et al., 2018), em Ciências Ambientais (ALAJMI; ALHAJI, 2018; WANG et al., 2018); Ciências da Decisão e Ciências Sociais (ALAJMI; ALHAJI, 2018; KOKOL et al.,2015), em Educação, Telecomunicação, Ergonomia, Sistemas de Controle e Automação (WANG et al., 2018).

Para Serenko (2013), os temas de estudos em GC apontam a existência de duas escolas de pensamento: uma centrada na tecnologia e que abrange estudos em áreas como Ciência da Computação, Sistemas de conhecimento, Engenharia, Inteligência Artificial e Tecnologia da informação), e outra, centrada no humano e no comportamento e que abrange estudos nas áreas: Ciências 
Cognitivas, Biblioteconomia e Informação, Filosofia, Psicologia, Gestão Estratégica, Pesquisa Operacional, Comportamento Organizacional, Recursos Humanos, Teoria Organizacional e Economia. Essa amplitude de áreas nas quais os estudos de GC estão presentes, indica o potencial de aplicação em outros campos, e, ao mesmo tempo, dificulta a convergência e avaliação das fronteiras do campo.

\section{ANÁLISE DOS RESULTADOS}

A configuração do campo da GC, a partir de estudos bibliométricos, foi obtida em três aspectos: periódicos, autores e interface com outras áreas. Quanto aos periódicos, o principal é o Journal of Knowledge Management. Além deste, há outros focados no tema, como: Knowledge Management Research \& Practice; International Journal of Knowledge Management; Knowledge \& Process Management; Electronic Journal of Knowledge Management e International Journal of Knowledge-Based Development. Os periódicos se concentram nas áreas: Informação e Comunicação (8 periódicos); Gestão/Estratégia (10) e Sistemas/Tecnologias (10).

Além da pluralidade de periódicos, é importante destacar que uma das características da área é a relevância de livros e publicações em conferências. Por exemplo, Kokol et al. (2015) avaliaram 10.599 fontes de informações, sendo 5111 publicadas em conferências, 4337 artigos em Journals, 496 revisões, 352 capítulos de livros e 86 livros. Quanto aos livros, a partir da década de 2000 a importância deles foi diminuída e o número dos artigos científicos apresentou aumento crescente (ALAJMI; ALHAJI, 2018).

Quanto aos autores que fundamentaram o campo, os pressupostos filosóficos foram atribuídos a Polaniy (dimensão tácita). A introdução dessa abordagem para a gestão foi consolidada por autores como Nonaka e Takeuchi, bem como Davenport e Prusak. Alguns autores mencionam também a influência de Peter Drucker para o estabelecimento de ideias da gestão do conhecimento, com a introdução do conceito de trabalhadores do conhecimento. Avançaram no campo da Estratégia a abordagem da capacidade absortiva do conhecimento, por meio das ideias de Cohen e Levinthal e as abordagens sobre capital 
intelectual, influenciadas por Sveiby. No início dos anos 2000, os autores Alavi e Leidner buscaram discutir o campo da GC, na ótica da área da Ciência da Informação. Como estudo crítico, destaca-se em 2002 o de Wilson (SERENKO, 2013). É importante salientar que, a partir de 2000 surgiu uma pluralidade de autores, a maioria deles publicando apenas um ou dois artigos, resultando em um elevado grau de diferenciação da disciplina (SERENKO, 2013).

Quanto à interface com outros campos do saber, é consenso que a área apresenta uma multidisciplinaridade. As disciplinas mais influentes foram: Ciência da Computação, Administração/Gestão/Organizações; Economia; Ciência da Informação/Sistemas de Informação; Comunicação; Ciência da Computação, Inteligência Artificial e Biblioteconomia. Serenko e Bontis (2013) apontam que esta é uma fragilidade da área, pois ela se apropria de conhecimentos de outras áreas.

Apesar da multidisciplinaridade, é necessário construir uma interdisciplinaridade a fim de que a área produza conhecimentos que the são próprios e não apenas tome emprestado de outras áreas e atinja uma robustez conceitual que não possui ainda. Avançar na direção da integração entre as áreas pode permitir uma visão do conhecimento além da funcional, predominante no campo (TZORTZAKI; MIHIOTIS, 2014).

De modo sucinto, os estudos bibliométricos demonstraram que o campo surgiu por volta dos anos 1970 e se consolidou a partir de algumas obras seminais. Ao longo da década de 1990, diversas áreas contribuíram com temáticas que foram incorporadas aos estudos de GC. A partir dos anos 2000 o número de publicações expandiu consideravelmente, tornando o campo multifacetado e desenvolvendo-se em duas vertentes, uma com publicações científicas em diversos Journals e outra em Conferências.

\section{CONSIDERAÇÕES FINAIS}

Os resultados desta pesquisa demonstram que a GC é um campo multidisciplinar e multifacetado, liderado por um conjunto de áreas como: Ciência da Computação, Administração/Gestão/Organizações; Economia; Ciência da Informação/Sistemas de Informação; Comunicação; Ciência da Computação, 
Inteligência Artificial e Biblioteconomia. Há pouca convergência entre as áreas que contribuem para a construção do campo, tanto em termos de autores, como em publicações.

O estudo demonstrou a relevância de estudos bibliométricos para avaliar a área de GC, embora com diferentes bases de dados. Serenko destacou-se como o autor que mais debateu o campo e o journal Knowledge of Management como o periódico que mais estudos bibliométricos publicou. Se por um lado, as bibliometrias elucidam diversos aspectos do campo, estes estudos consistem em levantamentos quantitativos, não possibilitando a abordagem de questões epistemológicas, sendo esta uma limitação do estudo.

Como contribuições desta pesquisa, destaca-se que os resultados propiciam um panorama evolutivo do campo e podem auxiliar pesquisadores, estudantes e profissionais que atuam ou desejam se inserir na área. Para o contexto brasileiro o estudo é importante para estimular o debate sobre o campo da GC, ainda pouco presente.

\section{REFERÊNCIAS}

AKHAVAN, P.; EBRAHIM, N. A.; FETRATI, M. A; PEZESHKAN, A. Major trends in knowledge management research: a bibliometric study.

Scientometrics, v. 107, n. 3, p. 1249-1264, 1 jun. 2016.

https://doi.org/10.1007/s11192-016-1938-x

ALAJMI, B.; ALHAJI, T. Mapping the Field of Knowledge Management:

Bibliometric and Content Analysis of Journal of Information \& Knowledge Management for the Period from 2002-2016. Journal of Information \&

Knowledge Management, v. 17, n. 03, p. 1-16, 30 jul. 2018.

https://doi.org/10.1142/S0219649218500272

ALAVI, M.; LEIDNER, D. E. Knowledge management and knowledge management systems: conceptual foundations and research issues. MIS Quarterly, v. 25, n. 1, pp. 107-136, 2011.

BONTIS, N.; SERENKO, A. A follow-up ranking of academic journals. Journal of Knowledge Management, v. 13, n. 1, p. 16-26, 20 fev. 2009.

CASSUNDÉ, F. R. S. A.; BARBOSA, M. A. C.; MENDONÇA, J. R. C. Entre revisões sistemáticas e bibliometrias: como tem sido mapeada a produção acadêmica em Administração no Brasil? Informação \& Informação, v. 23, n. 1, p. 311-334, 2018. 
COHEN, W.M.; LEVINTHAL, D. A. Absorptive capacity: A new perspective on learning and innovation. Administrative Science Quarterly, v. 35, n.1, p.128$152,1990$.

CURADO, C. M. M.; OLIVEIRA, M., MAÇADA, A. C. G. Mapping knowledge management authoring patterns and practices. African Journal of Business Management. v. 5, n. 22, p. 9137-9153, set., 2011 Disponível em: http://repositorio.pucrs.br/dspace/handle/10923/10247. Acesso em: 30 mar. 2020

DAVENPORT, T. H.; PRUSAK, L. Working Knowledge: How Organizations Manage what They Know. [S. I.] Harvard Business Press, 1998.

DRUCKER, P. Sociedade pós-capitalista. São Paulo: Pioneira, 1993.

DWIVEDI, Y. K; VENKITACHALAM, K.; SHARIF, A. M.; AL-KARAGHOULI, W.; WEERAKKODY, V. Research Trends in Knowledge Management: Analyzing the Past and Predicting the Future. Information Systems Management, v. 28, n. 1, p. 43-56, 10 jan. 2011.

ELLEGAARD, O.; WALLIN, J. A. The bibliometric analysis of scholarly production: How great is the impact? Scientometrics, v. 105, n. 3, p. 1809 1831, 2015.

GAVIRIA-MARIN, M.; MERIGO, J. M.; POPA, S. Twenty years of the Journal of Knowledge Management: a bibliometric analysis. Journal of Knowledge Management, v. 22, n. 8, p. 1655-1687, 4 jun. 2018. Disponível em: https://www.researchgate.net/publication/325564266_Twenty_years_of_the_Jo urnal_of_Knowledge_Management_a_bibliometric_analysis. Acesso em: 30 mar. $\overline{2} 020$

GOSWAMI, A. K.; AGRAWAL, R. K. A reflection on knowledge sharing research: patterns and trends. Vine Journal of information and Knowledge Management Systems, v. 48, n. 3, 352-372. 2018

GRANT, K. Knowledge Management, An Enduring but Confusing Fashion. Electronic Journal of Knowledge Management: EJKM; Reading, v. 9, n. 2, p. 117-131, abr. 2011.

HEIMERL, F.; HAN, Q.; KOCH, S.; ERTI, T. CiteRivers: Visual Analytics of Citation Patterns. IEEE Transactions on Visualization and Computer Graphics, v. 22, n. 1, p. 190-199, jan. 2016.

KHASSEH, A. A.; MOKHTARPOUR, R. Tracing the historical origins of knowledge management issues through referenced publication years spectroscopy (RPYS). Journal of Knowledge Management, v. 20, n. 6, p. 1393-1404, 10 out. 2016.

KOKOL, P.; ZLAHTIC, B.; ZLAHTIC, G.; ZORMAN, M.; PODGORELEC, V. Knowledge Management in Organizations - A Bibliometric Analysis of Research 
Trends. In: INTERNATIONAL CONFERENCE KMO, 10., Anais [...], 2015, v. 224, p. 3-14, Springer International Publishing, 2015.

LEE, M. R.; CHEN, T. T. Revealing research themes and trends in knowledge management: from 1995 to 2010. Knowledge-Based Systems, v. 28, p. 4758, abr. 2012.

MA, Z.; YU, K H. Research paradigms of contemporary knowledge management studies: 1998-2007. Journal of Knowledge Management, v. 14, n. 2, p. 175-189, abr. 2010.

MADEIRA, L. M. M.; VICK, T. E.; NAGANO, M. S. Directions of scientific literature in knowledge management from the perspective of their relationships with innovation, information and technology management. Transinformação, v. 25, n. 2, p. 167-174, ago. 2013. Disponível em: http://dx.doi.org/10.1590/S0103-37862013000200008. Acesso em: 30 mar. 2020

MUGNAINI, R.; DIGIAMPIETRI, L. A., MENA-CHALCO, J. P. Comunicação científica no Brasil (1998-2012): indexação, crescimento, fluxo e dispersão. Transinformação, v. 26, n. 3, p. 239-252, dez. 2014.

NONAKA, I.; TAKEUCHI, H. The Knowledge-creating Company: How Japanese Companies Create the Dynamics of Innovation. [S. I.] Oxford University Press, 1995.

NONAKA, I. A dynamic theory of Organizational Knowledge Creation. Organization Science, Organisation for economic co-operation and development. v. 5, n. 1, p. 14 - 37, fev. 1994.

QIU, J.; LV, H. An overview of knowledge management research viewed through the web of science (1993-2012). Aslib Journal of Information Management, v. 66, n. 4, p. 424-442, jul. 2014. Disponível em: https://doi.org/10.1108/AJIM-12-2013-0133. Acesso em: 30 mar. 2020

RAMY, A.; FLOODY, J.; RAGAB, M. M. F.; ARISHA, A. A scientometric analysis of Knowledge Management Research and Practice literature: $2003-$ 2015. Knowledge Management Research \& Practice, v. 16, n. 1, p. 66-77, jan. 2018. Disponível em: https://doi.org/10.1080/14778238.2017.1405776. Acesso em: 30 mar. 2020

SERENKO, A.; COX, R. A. K.; BONTIS, N.; BOOKER, L. D. The superstar phenomenon in the knowledge management and intellectual capital academic discipline. Journal of Informetrics, v. 5, n. 3, p. 2011

SERENKO, A. Meta-analysis of scientometric research of knowledge management: discovering the identity of the discipline. Journal of Knowledge Management, v. 17, n. 5, p. 773-812, 2013. 
SERENKO, A.; BONTIS, N.; BOOKER, L.; SADEDDIN, K.; HARDIE, T. A scientometric analysis of knowledge management and intellectual capital academic literature (1994-2008). Journal of Knowledge Management, v. 14, n. 1, p. 3-23, 23 fev. 2010.

SERENKO, A.; BONTIS, N. The intellectual core and impact of the knowledge management academic discipline. Journal of Knowledge Management, v. 17, n. 1, p. 137-155, 2013.

SERENKO, A.; BONTIS, N. Global ranking of knowledge management and intellectual capital academic journals: 2017 update. Journal of Knowledge Management, v. 21, n. 3, p. 675-692, mar. 2017.

SILVA, J. A. da; BIANCHI, M. de L. P. Cientometria: a métrica da ciência.

Paidéia, Ribeirão Preto, v. 11, n. 20, p. 5-10, 2001.

SVEIBY, K. E.; MARTINS, J. R. Gestão do conhecimento: as lições dos pioneiros. Global Brands, p. 1-6, 2006.

TSOUKAS, H.; VLADIMIROU, E. What is Organizational Knowledge? Journal of Management Studies, v. 38, n. 7, p. 973 - 993, nov. 2001.

TZORTZAKI, A. M.; MIHIOTIS, A. A Review of Knoledge Management Theory and Future directions. Knowledge and Process Management, v. 21, n. 1, p. 29-41, 2014.

UZUNBOYLU, H.; ERIŞ, H.; OZCINAR, Z. Results of a citation analysis of knowledge management in education. British Journal of Educational Technology, v. 42, n. 3, p. 527-538, 2011.

WALLACE, D. P.; VAN FLEET, C.; DOWNS, L. J. The research core of the knowledge management literature. International Journal of Information Management, v. 31, n. 1, p. 14-20, 1 fev. 2011.

WALTER, C.; RIBIÈRE, V. A citation and co-citation analysis of 10 years of KM theory and practices. Knowledge Management Research \& Practice, v. 11, n. 3, p. 221-229, 1 ago. 2013.

WALLIN, J. A. Bibliometric Methods: Pitfalls and Possibilities. Basic \& Clinical Pharmacology \& Toxicology 2005, v. 97, p. 261-275, 2005.

WANG, P.; ZHU, F. W.; SONG, H. Y.; HOU, J. H.; ZHANG, J. L. Visualizing the Academic Discipline of Knowledge Management. Sustainability, v. 10, n. 3, p. 682-710, mar. 2018.

WIIG, K. Knowledge Management: An Introduction and Perspective. Journal of Knowledge Management, v. 1, n. 1, p. 6-14, 1997. 
WILSON, T.D. The Nonsense of Knowledge Management. Information

Research, v. 8, n.1, paper144, 2002. Avaiable at http://informationr.net/ir/81/paper144.html.

ZUPIC, I.; ČATER, T. Bibliometric Methods in Management and Organization.

Organizational Research Methods, v. 18, n. 3, p. 429-472, 2015.

\title{
THE KNOWLEDGE MANAGEMENT FIELD FROM BIBLIOMETRIES
}

\begin{abstract}
Introduction: Knowledge Management is an interdisciplinary field, as a result, several bibliometric studies on the field have been published, seeking to identify its scope and contours. Objective: Map bibliometric studies in Knowledge Management in order to understand the boundaries of the field of study as well as the interfaces with other areas. Methodology: Qualitative, constituting a systematic analysis of bibliometric studies on knowledge management between 2008 and 2018. Results: Bibliometric studies have shown that the field emerged around the 1970s and was consolidated from some seminal works. Throughout the 1990s, several areas contributed to the approach of themes that were incorporated into KM studies. Since the 2000s, the number of publications has expanded considerably, making the field multifaceted and developing into two strands, one with scientific publications in several Journals and another in Conferences. Conclusions: KM's field presents itself as a broad field of study, with the contribution of the following areas: Computer Science, Administration/Management/ Organizations; Economy; Information Science/Information Systems; Communication; Computer Science, Artificial Intelligence and Library Science. There is little convergence between the areas that contribute to the construction of the field, both in terms of authors and publications.
\end{abstract}

Descriptors: Knowledge management. Bibliometrics. Information. Academic production.

\section{EL CAMPO DE LA GESTIÓN DEL CONOCIMIENTO DE LAS BIBLIOMETRÍAS}

\begin{abstract}
RESUMEN
Introducción: La Gestión del Conocimiento es un campo interdisciplinario, como resultado, se han publicado varios estudios bibliométricos en el campo, buscando identificar su alcance y contornos. Objetivo: Mapear estudios bibliométricos en Gestión del Conocimiento para comprender los límites del campo de estudio, así como las interfaces con otras áreas. Metodología: Cualitativa, que constituye un análisis sistemático de estudios bibliométricos sobre gestión del conocimiento entre 2008 y 2018. Resultados: Los estudios bibliométricos han demostrado que el campo surgió alrededor de la década de 1970 y se consolidó a partir de algunos trabajos fundamentales. A lo largo de la década de 1990, varias áreas contribuyeron al enfoque de los temas que se incorporaron a los estudios de GC. Desde la década de 2000, el número de publicaciones se ha expandido considerablemente, haciendo que el campo
\end{abstract}


tenga múltiples facetas y se convierta en dos líneas, una con publicaciones científicas en varias revistas y otra en conferencias. Conclusiones: El campo de KM se presenta como un amplio campo de estudio, con la contribución de las siguientes áreas: Informática, Administración/ Gestión/ Organizaciones; Economía; Ciencias de la información / Sistemas de información; Comunicación; Informática, Inteligencia Artificial y Biblioteconomía. Hay poca convergencia entre las áreas que contribuyen a la construcción del campo, tanto en términos de autores como de publicaciones.

Descriptores: Gestión del conocimiento. Bibliometría. Informacion. Producción académica.

Recebido em: 12/04/2019

Aceito em: 04/12/2019 